\title{
An experience of successful treatment of Rhodococcus equi infective endocarditis in a non-HIV patient with linezolid
}

\author{
Lim KY', Ab Rahman AK ${ }^{1 *}$ and Abdullah $\mathrm{FH}^{2}$ \\ ${ }^{1}$ Infectious Disease Unit, Department of Medicine, Hospital Sultanah Nur Zahirah, Kuala Terengganu, Terengganu, Malaysia \\ ${ }^{2}$ Microbiology Unit, Hospital Sultanah Nur Zahirah, Kuala Terengganu, Terengganu, Malaysia
}

\begin{abstract}
Rhodococcus equi infection is an uncommon infection in the immunocompetent patient. It is a common zoonotic infection, with lung being the commonest primary site of infection. This organism is commonly misidentified as contaminant as it resembles diptheroids. Once recognised, the treatment requires combination of at least two different classes of antibiotics with a prolonged duration. Here, we report a case of rhodococcus equi infective endocarditis in a serologically HIV negative patient with no exposure to farm animals, who was resistant to many common antibiotics and successfully treated with linezolid.
\end{abstract}

\section{Introduction}

Rhodococcus equi is an aerobic gram positive organism which is a common cause of disease of the animals. Being a well-known pathogen in the veterinary medicine field, it was only after the 1960s that human infection cases were reported, with the first human infection reported in 1967 [1]. In human, it usually affects individuals with impaired host immunity such as in human immunodeficiency virus [2]. Infection among immunocompetent person is extremely rare. Lungs are commonly reported to be the primary site of infection for Rhodococcus equi. Other less commonly reported sites of infection were gastrointestinal, pericarditis, meningitis and mastoiditis with other cases of reported liver, kidney and psoas muscle abscesses [3]. It is unusual for rhodococcus to cause an infective endocarditis [4]. Here, we report a case of refractory rhodococcal infective endocarditis in an immunocompetent intravenous drug user.

\section{Case report}

A 32 year old gentleman with chronic hepatitis $C$ who was an ex intravenous drug user presented in early November 2013 with fever of two weeks duration. There was no symptoms to suggest respiratory tract, gastrointestinal or urinary tract infection. He denied any history of contact with other ill individuals, no history of recent travel or contact with livestock.

On initial examination, he was febrile with temperature of $39^{\circ} \mathrm{C}$ and other vital signs were stable. Cardiovascular examination revealed presence of TR and MR murmur but he had no peripheral stigmata of infective endocarditis. He has few evidences of stigmata of chronic liver disease like mild splenomegaly and mild thrombocytopenia. Other physical examination was unremarkable, his HIV serological test which was obtained after an informed consent was negative.

Initial laboratory investigation revealed TWCC of $13,000\left(10^{9} / \mathrm{L}\right)$ and raised C-reactive protein. Liver function profile and renal function were normal. There was no laboratory evidence of cirrhosis or coagulopathy. Chest $\mathrm{x}$ ray showed no lung lesions. A transthoracic echocardiogram revealed vegetation measuring $0.4 \mathrm{~cm}^{2}$ at the pulmonary valve with presence of moderate mitral regurgitation and tricuspid regurgitation. Ejection fraction was $74 \%$.
His blood culture grew Rhodococcus equi. The organism was susceptible to gentamicin, imipenem, meropenem, rifampicin, and erythromycin. In this hospital, disc diffusion method was used as a standard method of test. He was subsequently started on intravenous erythromycin 500mg every 6 hours, coupled with IV gentamicin and oral rifampicin $600 \mathrm{mg}$ daily.

In view of persistent fever, blood cultures were repeated several times and all grew rhodococcus equi. Imipenem and vancomycin were added on later. The blood culture taken six weeks after the initiation of antibiotics showed organism that resistant to all current antibiotics including vancomycin and carbapenem. However it was still susceptible to amikacin. No other foci of infection were detected. A repeat chest $\mathrm{x}$ ray showed no new pneumonic patch. He was also referred to cardiothoracic surgeon in view of persistent fever and valvular vegetation, however was managed conservatively. The low grade fever persisted after few weeks of combination antibiotics. His clinical condition did not improve much. His serial C-reactive proteins (CRP) were persistently elevated but other blood parameters were otherwise stable.

The blood culture which was repeated after seven weeks of treatment still grew the same organism. At this time, a susceptibility test using disc diffusion towards linezolid was carried out and the organism was found to be susceptible to it. An intravenous linezolid $600 \mathrm{mg} 12$ hourly was started. With the introduction of linezolid, his general condition improved remarkably and the fever settled after 10 days of adding in linezolid. CRP became normalised after 1 week of therapy. A repeat blood culture was negative. Subsequently, all other antibiotics were stopped and he was on linezolid monotherapy. As he

Correspondence to: $\mathrm{Ab}$ Rahman $\mathrm{AK}$, Infectious Disease Unit, Department of Medicine, Hospital Sultanah Nur Zahirah, Kuala Terengganu, Terengganu, Malaysia, E-mail: drfha_drakar@yahoo.com

Key words: Rhodococcus equi, infective endocarditis, resistant, linezolid, non-HIV Received: October 08, 2017; Accepted: October 25, 2017; Published: October 27,2017 
insisted for discharge, we discharged him after being afebrile for more than 3 days with oral linezolid and planned for weekly monitoring of clinical symptoms and full blood count at the clinic.

He was seen in the clinic a week later and had been afebrile since discharge. A repeated outpatient transthoracic echocardiogram showed severe mitral regurgitation but the previously seen vegetation at the pulmonary valve has disappeared. He was seen again at the clinic on day 38 of linezolid. He had been afebrile, however complained of lethargic. A complete blood count noted pancytopenia with haemoglobin of 6.3 $\mathrm{g} / \mathrm{dl}$, white cell count of $2,300\left(10^{9} / \mathrm{L}\right)$ and platelet of $41,000\left(10^{9} / \mathrm{L}\right) . \mathrm{He}$ received 2 units of packed cell transfusion. In view of symptomatic anaemia together with the absence of active inflammation, we decided to stop linezolid. He refused for a cardiothoracic referral for the correction of severe valvular insufficiency. A repeat complete blood count a week later showed recovery of the cell counts to near normal value (Table 1 ).

Three months after stopping linezolid he remained well until one day he presented to the Emergency Department with acute onset of breathlessness. Clinical and radiological examinations revealed evidence of acute pulmonary oedema most likely secondary to severe valvular heart lesions. Subsequently he developed a sudden cardiac arrest and later succumbed to the illness shortly after. A post mortem examination was offered but refused by the wife.

Detailed history revealed that he had no history of recurrent fever. In fact his general condition has improved and he had gained $4 \mathrm{~kg}$ ever since he was last discharged from the hospital.

\section{Discussion}

Rhodococcus equi infection rate in an immunocompetent patient had been reported to be only $11 \%$. A higher rate of infection in the immunocompromised group have been reported; $50-55 \%$ in patients with HIV and 20-25\% in non HIV immunocompromised patients [5].

As a zoonotic infection, exposure to farm animals plays a role, but it is not mandatory as most patients may not have the history, as in our patient. It can also be acquired from contaminated soil via inhalation, or inoculation into wound or mucous membrane or through the alimentary tract via ingestion [6]. Other modes of transmission are still not well understood, and that include human colonization and human to human transmission.

There had not been many reported cases of Rhodococcus equi being the causative organism of infective endocarditis. In the retrospective study which was carried out in Spain, only one patient out of 54 patients with infective endocarditis, was found to have Rhodococcus equi as the causative microorganism [7]. Matsushita et al. also reported a case of Rhodococcus equi infective endocarditis which was treated successfully with vancomycin and ciprofloxacin [8].

Based on CLSI M45 susceptibility testing for Rhodococcus spp, this organism was in the same group and shared the same testing profile as for Corynebacterium sp. [9] The organism is typically sensitive to vancomycin, erythromycin, aminogylcosides, rifampicin and imipenem but intrinsically resistant to penicillin and cephalosporin groups of antibiotics. There had been no guideline published for treatment of Rhodococcus equi infection, with no standardised antibiotic regimen [4]. Based on expert opinions consensus, recommendation of treatment had been the utilization of at least two types of antibiotics from different classes for a minimum of two months duration [3], Relapse is quite common and therefore for full eradication of the infection and prevention of disease relapse, a long term course of maintenance antibiotic treatment is deemed necessary especially in an immunocompromised patient [4]. Emergence of newer class of antibiotics had given rise to alternative choice of treatment to erythromycin and rifampicin, namely the newer macrolides, which are azithromycin and clarithromycin [10]. However, our patient did not respond to macrolide, aminoglycoside, rifampicin and imipenem due to him having a multidrug resistant organism; which some of them developed during the course of treatment.

Linezolid is an orally active drugs belonging to the oxazolidones group. Data on the efficacy of linezolid in the treatment of Rhodococcus equi infection is still lacking and little is known on its efficacy. Data on in vivo effectiveness of linezolid is sparse [11].

Even though linezolid is one of the antibiotic panels recommended for susceptibility testing for gram-positive bacilli organism including Corynebacterium sp., the test was not offered routinely in our hospital. During this time, the use of linezolid beyond two weeks duration was not approved by US Food and Drug Authority due to its significant but reversible marrow toxicity; therefore making it a less attractive choice of antibiotic to treat infective endocarditis.

Despite this limitation, there are few case reports citing the successful use of extended duration of linezolid in treating difficult infections other than endocarditis. Russo et al. had reported successful treatment of refractory Rhodococcus equi retroperitoneal abscess with combination of linezolid and tigecycline followed by linezolid/ meropenem combination for a total of 18 weeks. However, the haematological effect of this treatment regimen was not stated [4]. Munoz et al. reported the first successful case of a post heart transplant rhodococcal infection which was successfully treated with five months of linezolid monotherapy [12]. Again, they did not mention the haematological effect of the treatment. Our patient had been successfully treated with extended duration of linezolid but complicated with marrow suppression which leads to termination of treatment at day 38. The effect of marrow suppression even though severe; was actually reversible. We continued to monitor for a complete marrow recovery as well as for any sign of relapse. Unfortunately though, he presented again to the hospital with features of acute pulmonary oedema and subsequently he had a fatal cardiac arrest. There was no evidence to suggest a relapsed infection. His complete blood count also showed recovery of all cell lines.

In summary, Rhodococcus equi infection in immunocompetent patients is not common, and rhodococcal infective endocarditis is even rarer. Experience in treating this condition is understandably very limited. Whilst most cases of rhodococcal infections can be treated successfully with combination of the first line antibiotics, in small percentage of patients with a multidrug- resistant strain it may require

Table 1. Profile of blood counts before, after initiation and after completion of linezolid

\begin{tabular}{|c|c|c|c|c|}
\hline Full blood counts & Before starting linezolid & Day 38 of linezolid & 6 weeks after stopping linezolid & $\begin{array}{c}\text { Three months post treatment } \\
\text { completion }\end{array}$ \\
\hline Hemoglobin $(\mathrm{g} / \mathrm{L})$ & 8.0 & 4.7 & 8.0 & 9.2 \\
\hline Total White cells $\left(10^{9} / \mathrm{L}\right)$ & 2.6 & 4.0 & 4.1 & 8.7 \\
\hline Plaetelet $\left(10^{9} / \mathrm{L}\right)$ & 77 & 66 & 47 & 74 \\
\hline
\end{tabular}


a different agent. Linezolid even though is an effective alternative agent, but is compounded by bone marrow toxicity effect. In treating a refractory rhodococcal infective endocarditis such like this; and in the absence of a safer alternative, linezolid should be used with great caution. This patient had been successfully treated with linezolid despite been complicated with marrow suppression. The marrow had subsequently recovered to the near baseline value after stopping the offending drug over few weeks.

\section{References}

1. Prescott JF (1991) Rhodococcus equi: an animal and human pathogen. Clin Microbiol Rev 4: 20-34. [Crossref]

2. Torres-Tortosa M (2003) Prognosis and Clinical Evaluation of Infection Caused by Rhodococcus equi in HIV-Infected Patients. Chest $J 123$.

3. Kamboj M, Kalra A, Kak V (2005) Rhodococcus equi brain abscess in a patient without HIV. J Clin Pathol 58: 423-425. [Crossref]

4. Russo G, Lichtner M, Carnevalini M, Mascellino MT, Mengoni F, et al. (2010) Primary retroperitoneal abscesses due to Rhodococcus equi in a patient with severe nephrotic syndrome: successful antibiotic treatment with linezolid and tigecycline. Int $J$ Infect Dis 14: e533-5. [Crossref]
5. Hayes D Jr, Diaz-Guzman E, Hoopes CW (2011) Rhodococcus equi infection after lung transplantation. Respir Care 56: 1605-1607. [Crossref]

6. Weinstock DM, Brown AE (2002) Rhodococcus equi: an emerging pathogen. Clin Infect Dis 34: 1379-1385. [Crossref]

7. Roca B, Marco JM (2007) Presentation and outcome of infective endocarditis in Spain: a retrospective study. Int J Infect Dis 11: 198-203. [Crossref]

8. Matsushita H, Hanayama N, Hobo K, Kuba K, Takazawa A (2010) Infectious endocarditis caused by Rhodococcus equi. Ann Thorac Surg 89: 957-959. [Crossref]

9. CLSI (2006) Methods for antimicrobial dilution and disk susceptibility testing of infrequently isolated or fastidious bacteria, M45-A vol 26 No. 19 Clinical and Laboratory Standards Institute, Wayne, PA.

10. Cisek AA, Rzewuska M, Witkowski L, Binek M (2014) Antimicrobial resistance in Rhodococcus equi. Acta Biochim Pol 61: 633-638. [Crossref]

11. Weinstock DM1, Brown AE (2002) Rhodococcus equi: an emerging pathogen. Clin Infect Dis 34: 1379-1385. [Crossref]

12. Muñoz P1, Palomo J, Guinea J, Yañez J, Giannella M, et al. (2008) Relapsing Rhodococcus equi infection in a heart transplant recipient successfully treated with long-term linezolid. Diagn Microbiol Infect Dis 60: 197-199. [Crossref]

Copyright: (C2017 Lim KY. This is an open-access article distributed under the terms of the Creative Commons Attribution License, which permits unrestricted use, distribution, and reproduction in any medium, provided the original author and source are credited. 